\title{
Response of photoelectron peaks in the Martian ionosphere to solar EUV/X-ray irradiance
}

\author{
XiaoShu Wu' ${ }^{1}$, Jun Cui ${ }^{2} 3^{*}$, YuTian Cao', WeiQin Sun², Qiong Luo ${ }^{4}$, and BinBin Ni4 \\ ${ }^{1}$ National Astronomical Observatories, Chinese Academy of Sciences, Beijing 100012, China; \\ 2Planetary Environmental and Astrobiological Research Laboratory (PEARL), School of Atmospheric Sciences, Sun Yat-sen University, Zhuhai Guangdong \\ 519082, China; \\ ${ }^{3}$ Chinese Academy of Sciences Center for Excellence in Comparative Planetology, Hefei 230026, China; \\ ${ }^{4}$ Department of Space Physics, School of Electronic Information, Wuhan University, Wuhan 430074, China

\section{Key Points:} \\ - The photoelectron peaks near $27 \mathrm{eV}$ and $500 \mathrm{eV}$ are investigated, related to HeII $30.4 \mathrm{~nm}$ ionization and O Auger ionization \\ - The $500 \mathrm{eV}$ peak intensity increases with increasing solar irradiance much more rapidly than the 27 eV peak intensity \\ - The observation is consistent with a larger variability in solar radiance at shorter wavelengths
}

Citation: Wu, X. S., Cui, J., Cao, Y. T., Sun, W. Q., Luo, Q., and Ni, B. B. (2020). Response of photoelectron peaks in the Martian ionosphere to solar EUV/X-ray irradiance. Earth Planet. Phys., 4(4), 390-395. http://doi.org/10.26464/epp2020035

\begin{abstract}
An important population of the dayside Martian ionosphere are photoelectrons that are produced by solar Extreme Ultraviolet and X-ray ionization of atmospheric neutrals. A typical photoelectron energy spectrum is characterized by a distinctive peak near 27 eV related to the strong solar HeII emission line at $30.4 \mathrm{~nm}$, and an additional peak near $500 \mathrm{eV}$ related to O Auger ionization. In this study, the extensive measurements made by the Solar Wind Electron Analyzer on board the recent Mars Atmosphere and Volatile Evolution spacecraft are analyzed and found to verify the scenario that Martian ionosphere photoelectrons are driven by solar radiation. We report that the photoelectron intensities at the centers of both peaks increase steadily with increasing solar ionizing flux below $90 \mathrm{~nm}$ and that the observed solar cycle variation is substantially more prominent near the O Auger peak than near the HeII peak. The latter observation is clearly driven by a larger variability in solar irradiance at shorter wavelengths. When the solar ionizing flux increases from $1 \mathrm{~mW} \cdot \mathrm{m}^{-2}$ to $2.5 \mathrm{~mW} \cdot \mathrm{m}^{-2}$, the photoelectron intensity increases by a factor of 3.2 at the HeII peak and by a much larger factor of 10.5 at the $\mathrm{O}$ Auger peak, both within the optically thin regions of the Martian atmosphere.
\end{abstract}

Keywords: Mars; ionosphere; photoelectron; solar irradiance; MAVEN

\section{Introduction}

Mars exhibits a well-defined ionosphere on the dayside that is produced primarily by solar Extreme Ultraviolet (EUV) and X-ray ionization (Withers, 2009; Haider and Mahajan, 2014). One of the extensively studied components of the dayside Martian ionosphere is photoelectrons with energies typically above several eV (Coates et al., 2011). The energy distribution of these non-thermal electrons is usually characterized by (1) several distinctive spectral peaks near $27 \mathrm{eV}$ connected to the strong solar HeII emission line at $30.4 \mathrm{~nm},(2)$ an apparent knee at 60-70 eV associated with the rapid drop in solar flux at wavelengths shorter than $17 \mathrm{~nm}$, and (3) an additional peak near $500 \mathrm{eV}$ produced by (inner shell) $\mathrm{O}$ Auger ionization of atmospheric neutrals (e.g. Mantas and Hanson, 1979; Shutte et al., 1989; Frahm et al., 2006a; Sakai et al., 2015; Peterson et al., 2016).

Correspondence to: J. Cui, cuijun7@mail.sysu.edu.cn

Received 14 FEB 2020; Accepted 10 APR 2020.

Accepted article online 05 JUN 2020.

(C) 2020 by Earth and Planetary Physics.
Photoelectrons are important in at least two aspects: (1) they heat ionospheric thermal electrons efficiently via Coulomb collisions and cause impact ionization of atmospheric neutrals, hence affecting both the local energy balance and ionization balance (e.g. Matta et al., 2014; Sakai et al., 2016; Cui J et al., 2018); (2) they are frequently observed at locations where their local production is not expected, and hence they are in practice used as a diagnostic of the ambient magnetic field topology (e.g. Coates et al., 2015; Xu SS et al., 2017; Adams et al., 2018; Cao YT et al., 2020a). An interesting application of the latter is the occasional observation of photoelectrons on the nightside, indicative of closed magnetic loops that connect the dayside and nightside of the Martian ionosphere (e.g. Xu SS et al., 2016a; Cao YT et al., 2020b).

Numerous photoelectron spectra have been accumulated over the past few decades by a number of instruments such as the Magnetometer/Electron Reflectometer (MAG/ER) on board the Mars Global Surveyor (MGS) (Mitchell et al., 2001), the Electron Spectrometer as a part of the Analyzer of Space and Energetic Atoms on board the Mars Express (Frahm et al., 2006b), and more recently the Solar Wind Electron Analyzer (SWEA) on board the 
Mars Atmosphere and Volatile Evolution (MAVEN) spacecraft (Mitchell et al., 2016). The large data set currently available allows variations of the photoelectron energy spectrum to be investigated systematically.

Based on MGS MAG/ER data, Trantham et al. (2011) found that most of the variation of photoelectron intensity was driven by solar cycle variation; variation in the background atmosphere also played a noticeable role, especially during global dust storms. A similar solar cycle variation was reported by Xu SS et al. (2016b) also based on MGS MAG/ER data; they found further that the photoelectron intensity was roughly independent of solar zenith angle (SZA). Using MAVEN SWEA data, Wu XS et al. (2019a) analyzed the shape of the photoelectron energy spectrum, revealing systematic trends with altitude and SZA. The SWEA data are unique among existing measurements near Mars in that they provide the only opportunity so far to explore characteristics of photoelectrons down to the ionospheric peak altitude.

In this study, we examine further the energy dependent solar cycle variation of photoelectron intensity, using as two proxies the intensities at the HeII peak near $27 \mathrm{eV}$ and the O Auger peak near $500 \mathrm{eV}$. We show that the $\mathrm{O}$ Auger peak provides a better diagnostic of the solar EUV/X-ray input at Mars, as fully expected by the solar driven scenario. The present study could be viewed as an extrapolation of existing investigations at high altitudes (e.g. Trantham et al., 2011; Xu SS et al., 2016b) towards regions below the ionopause (e.g. Vogt et al., 2015). The parameterized solar cycle variations inferred from the SWEA data are well suited for future comparisons with kinetic model results obtained under different solar EUV/X-ray inputs (e.g. Liemohn et al., 2003, 2006; Xu SS et al., 2015; Xu SS and Liemohn, 2015; Sakai et al., 2015; Peterson et al., 2016).

\section{Observations}

For the purpose of this study, we include in our analysis the SWEA photoelectron spectra acquired over the dayside of Mars with SZA below $85^{\circ}$ and up to a maximum altitude of $500 \mathrm{~km}$. A total of 2.7 million spectra are available for this study, from the arrival of MAVEN at Mars on 22 September 2014 to 14 February 2018 (Jakosky et al., 2015). Only the omnidirectional photoelectron intensities averaged over the SWEA instrument field of view are used. The energy resolution of a SWEA spectrum is typically $17 \%$ according to Mitchell et al. (2016). All spectra have been properly corrected for spacecraft charging using potentials derived from MAVEN Suprathermal and Thermal Ion Composition measurements (McFadden et al., 2015).

For illustrative purposes, we display in Figure 1 the SWEA photoelectron energy spectra acquired during MAVEN orbit \#1061 as a function of time from periapsis. Also provided are the variations of the altitude, SZA, and latitude along the spacecraft trajectory below $500 \mathrm{~km}$. The solar EUV/X-ray flux is $1.62 \mathrm{~mW} \cdot \mathrm{m}^{-2}$ when integrated over the wavelength range from $0.5 \mathrm{~nm}$ to the $\mathrm{CO}_{2}$ ionization threshold of $90 \mathrm{~nm}$ based on the level 3 solar spectral model at Mars (Thiemann et al., 2017) calibrated with MAVEN Extreme UItraviolet Monitor (EUVM) band irradiance data (Eparvier et al., 2015). Such a flux is referred to as "the solar ionizing flux" throughout the rest of the paper.
In Figure 1, both the HeII peak and the knee are clearly observed whereas the O Auger peak is seen only marginally due to substantially lower signal-to-noise ratios at such high energies (Mitchell et al., 2016). Three representative one-dimensional cuts of the twodimensional spectrum are compared in the bottom row of Figure 1 for $391.7 \mathrm{~km}, 196.8 \mathrm{~km}$, and $133.5 \mathrm{~km}$, respectively, revealing the absence of the HeII and O Auger peaks at high altitudes but the maintenance of both peaks at low altitudes. There is also evidence for a declining intensity at the two peaks as the altitude declines towards the periapsis, a feature that is clearly attributable to the reduced solar ionizing flux near and below the ionospheric peak (e.g. Hantsch and Bauer, 1990; Morgan et al., 2008; Yao MJ et al., 2019). The presence of a photoelectron boundary, where the HeII peak switches between visible and invisible, is evident near $280 \mathrm{~km}$ during the inbound portion of the orbit and near $240 \mathrm{~km}$ during the outbound portion (Garnier et al., 2017; Han QQ et al., 2019). The typical uncertainty in photoelectron intensity is at most several percent near the HeII peak and not more than $10 \%$ near the O Auger peak.

\section{Solar Cycle Variations of Photoelectron Peaks}

The entire available MAVEN SWEA data set is ideal for exploring quantitatively the solar control of the spectral peaks seen in the photoelectron energy distribution in the dayside Martian ionosphere, which we present in this section.

In Figures 2 and 3, we show the SWEA-based photoelectron intensities at the centers of the HeII and O Auger peaks near $27 \mathrm{eV}$ and $500 \mathrm{eV}$, respectively, as a function of the EUVM-based solar ionizing flux. In each panel, the displayed variation is constructed by interpolating to the desired altitude the measured intensities during either the inbound or outbound MAVEN orbit. The variations at different altitudes are compared as indicated in the figure legend, all revealing the expected trend of increasing photoelectron intensity with increasing solar activity, as already noted by Trantham et al. (2011) and Xu SS et al. (2016a) for a fixed altitude of $400 \mathrm{~km}$ using the MGS MAG/ER data. For the O Auger peak, the measured intensity varies substantially from orbit to orbit, partially due to the contamination of Solar Wind electrons well above the photoelectron knee, but a clear solar cycle trend can still be spotted. The photoelectron intensities displayed in both figures are obtained by identifying the energy closest to $27 \mathrm{eV}$ for the HeII peak and to $500 \mathrm{eV}$ for the $\mathrm{O}$ Auger peak, rather than by interpolating the measured intensities to exactly the required energies. This is to avoid the unrealistic values of photoelectron intensity often encountered in numerical interpolation, especially for the high energy intensities which are faint and subject to large uncertainties. Such a procedure implies an undesired energy shift between different spectra but this shift is in general smaller than the SWEA energy resolution of $\sim 4.6 \mathrm{eV}$ near the HeII peak and $85 \mathrm{eV}$ near the $\mathrm{O}$ Auger peak. Note also that in their evaluation of the solar cycle variation of photoelectron intensity, Trantham et al. (2011) took into account the attenuation of the incident solar irradiance along a limb path for a fixed SZA, but we choose not to correct for this effect as Xu SS et al. (2016b) showed that the photoelectron intensity was better correlated directly with the solar EUV/X-ray flux without SZA factored in. 

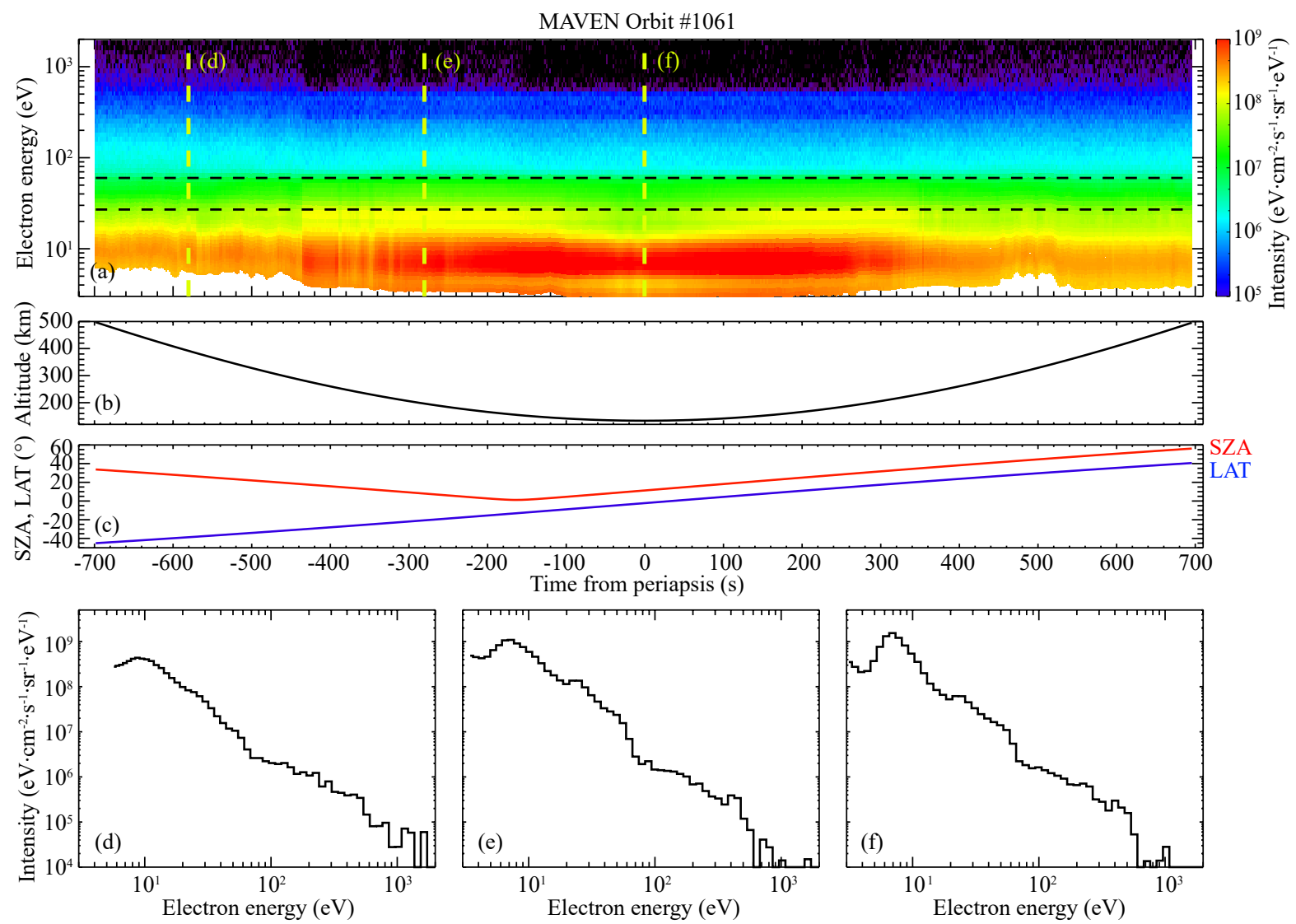

Figure 1. (a) SWEA photoelectron energy spectra as a function of time from periapsis, acquired during MAVEN orbit \#1061; the horizontal dashed lines indicate the locations of the HeII and O Auger peaks. (b) Spacecraft altitude. (c) SZA and latitude (LAT). Three representative onedimensional cuts of the photoelectron energy distribution, as indicated by the vertical dashed lines in Panel (a), are provided in Panels (d), (e), and (f) for comparison.

Of special interest is the observation of a substantially more prominent response of photoelectron intensity to varying solar ionizing flux at the O Auger peak than at the HeII peak. The solar cycle variations in Figures 2 and 3 could each be reasonably described by a power law in the form of $l_{\mathrm{e}}(E) \propto F_{i}^{a(E)}$ where $E$ and $l_{\mathrm{e}}$ are the photoelectron energy and omnidirectional intensity, $F_{i}$ is the solar ionizing flux, and $a$ is the energy-dependent power index used to parameterize the observed solar cycle variation. This functional form reflects properly the desired limiting behavior of zero intensity when the solar EUV/X-ray irradiance is turned off. The best-fit parameters, $a$, are listed in Table 1 for reference, which are on average 1.28 for the HeII peak and 2.57 for the O Auger peak. These values further indicate that as the solar ionizing flux increases from $1 \mathrm{~mW} \cdot \mathrm{m}^{-2}$ to $2.5 \mathrm{~mW} \cdot \mathrm{m}^{-2}$, the photoelectron intensity at the HeII peak increases by a factor of 3.2 whereas the intensity at the O Auger peak increases by the much larger factor of 10.5 . It is also interesting to note from Table 1 that the solar cycle variation of photoelectron intensity appears to be altitude independent, which is also an expected result within the optically thin regions of the atmosphere. At altitudes near or below the ionospheric peak (e.g. Hantsch and Bauer, 1990; Morgan et al., 2008; Yao MJ et al., 2019), some dependence on altitude is foreseen, connected to the change in optical depth. We also caution that the best-fit power laws have been obtained over the range of solar ionizing flux from $0.7 \mathrm{~mW} \cdot \mathrm{m}^{-2}$ to $2.5 \mathrm{~mW} \cdot \mathrm{m}^{-2}$ and cannot be extrapolated to arbitrarily high and low incident solar EUV/X-ray levels.

Within the optically thin regions of the Martian ionosphere, the photoelectron intensity should be directly proportional to the solar EUV/X-ray flux (e.g. Wu XS et al., 2019b). One may then expect a linear increase in photoelectron intensity with increasing solar ionizing flux, but the best-fit functional form that we obtain suggests a more steepened increase. This finding is linked to the fact that the solar ionizing flux at shorter wavelengths varies more substantially with solar activity than at longer wavelengths. Put another way, the enhancement in the integrated solar ionizing flux as solar activity increases is not proportionally distributed over the wavelength range involved in photoelectron production; a more prominent enhancement is observed at wavelengths responsible for the $\mathrm{O}$ Auger peak than at wavelengths responsible for the HeII peak. Meanwhile, both HeII and O Auger photoelectrons may also be produced via "secondary" impact ionization of atmospheric neutrals by more energetic photoelectrons, which would act to enhance the observed trend in photoelectron intensity with solar activity.

For comparison, Trantham et al. (2011), using as a proxy the conventional $10.7 \mathrm{~cm}$ solar radio index at the Earth adjusted for the Sun-Mars distance, reported that photoelectron intensity varied with solar EUV irradiance in a roughly linear way. Clearly, the observed correlation between photoelectron intensity and solar irra- 

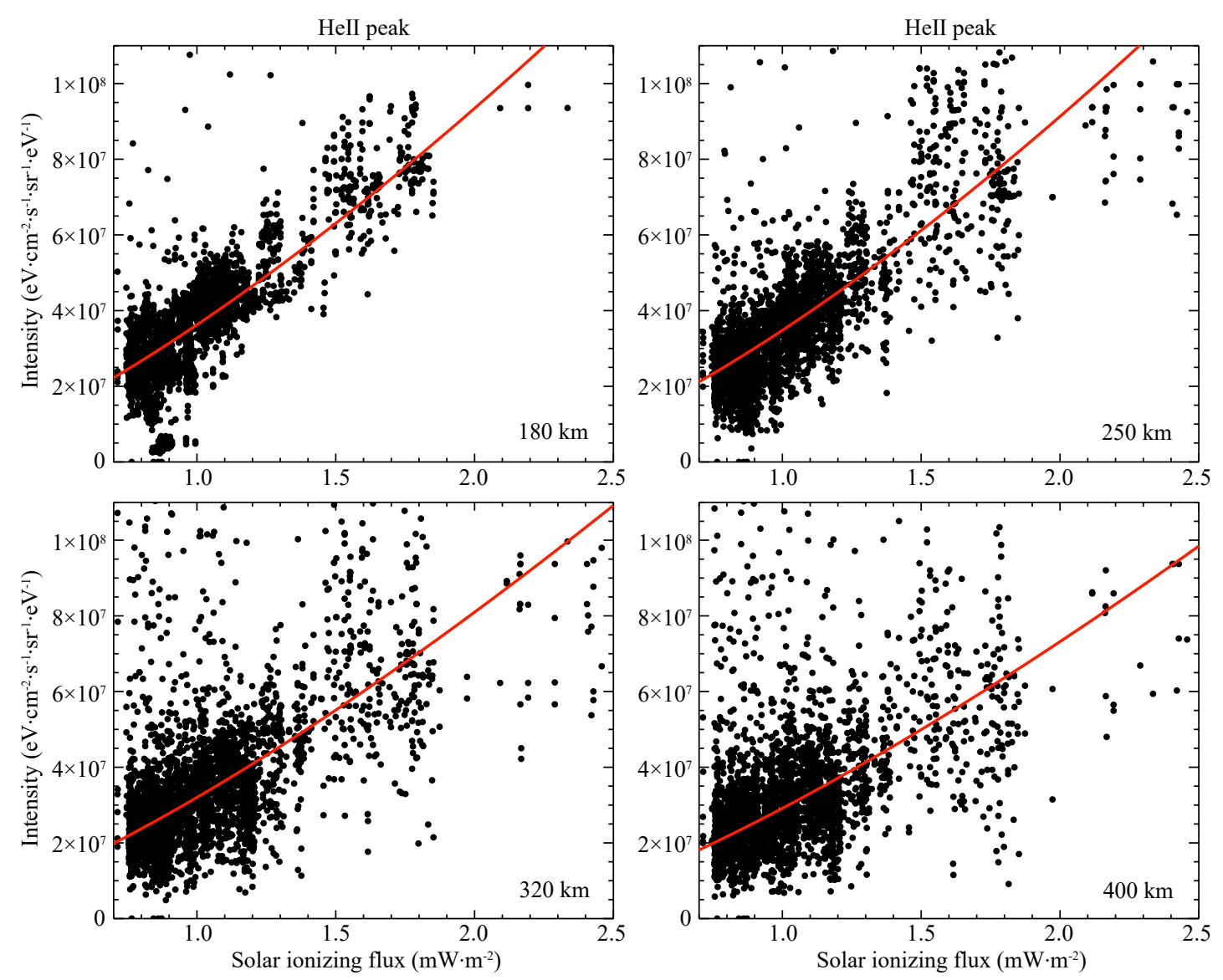

Figure 2. Solar cycle variation of photoelectron intensity at the center of the HeII peak, at four representative altitudes as indicated in the figure legend.

diance depends somewhat on the actual solar EUV/X-ray proxy used. With the direct monitoring of solar EUV/X-ray flux at Mars facilitated by the EUVM instrument, MAVEN data obviously provide a more appropriate sample for exploring solar cycle variations of a range of Martian ionospheric parameters including the photoelectron intensity. In addition, we note that the scatterings in the observed solar cycle variation, as displayed in Figures 2 and 3, are fairly large. While the power law relation does provide the best-fit to the observations, other functional forms including a linear one are probably acceptable. Within the context of this study, of more importance is the energy dependent solar cycle variation of photoelectron intensity, as clearly suggested by the data; this finding is independent of the detailed functional forms used for parameterizing the observations.

\section{Concluding Remarks}

Photoelectrons are an important population of the dayside Martian ionosphere and are produced by solar EUV/X-ray ionization of atmospheric neutrals (e.g. Mantas and Hanson, 1979; Shutte et al., 1989; Frahm et al., 2006a; Sakai et al., 2015). Such a solar driven scenario, though established for decades, has not been empirically verified in detail, mainly due to limited in-situ measurements of photoelectrons, especially below the Martian ionopause. The studies of Trantham et al. (2011) and Xu SS et al. (2016b) did report evidence for solar cycle variation of photoelectron intensity but their analyses were performed at a fixed altitude of $400 \mathrm{~km}$ due to the limited coverage of MGS MAG/ER data. The recent MAVEN SWEA measurements (Mitchell et al., 2016) have provided a unique opportunity to explore more thoroughly the solar control of photoelectron intensity, not only by virtue of extensive measurements of the photoelectron energy distribution down to regions well below the ionospause (Mitchell et al., 2016) but also by virtue of their simultaneous monitoring of the solar EUV/X-ray flux right at Mars (Eparvier et al., 2015).

In this study, we analyze a total of 2.7 million SWEA photoelectron energy spectra accumulated over three years and a half, from which we are able to identify a strong solar cycle variation of photoelectron intensity at different energies. We focus specifically on two energies, $27 \mathrm{eV}$ as the center of the HeII peak and $500 \mathrm{eV}$ as the center of the $O$ Auger peak. Our analysis reveals that despite the observed increase in photoelectron intensity with increasing solar ionizing flux at both peaks, this trend is clearly less prominent at the HeII peak than at the O Auger peak, as expected by a larger variability in solar irradiance at shorter wavelengths. The solar cycle variation of photoelectron intensity could be reasonably described by a power law relation, predicting that over the range of solar ionizing flux from $1 \mathrm{~mW} \cdot \mathrm{m}^{-2}$ to $2.5 \mathrm{~mW} \cdot \mathrm{m}^{-2}$, the photoelectron intensity increases by a factor of 3.2 at the HeII peak and by the much larger factor of 10.5 at the O Auger peak, both within the optically thin regions of the Martian atmosphere. The 

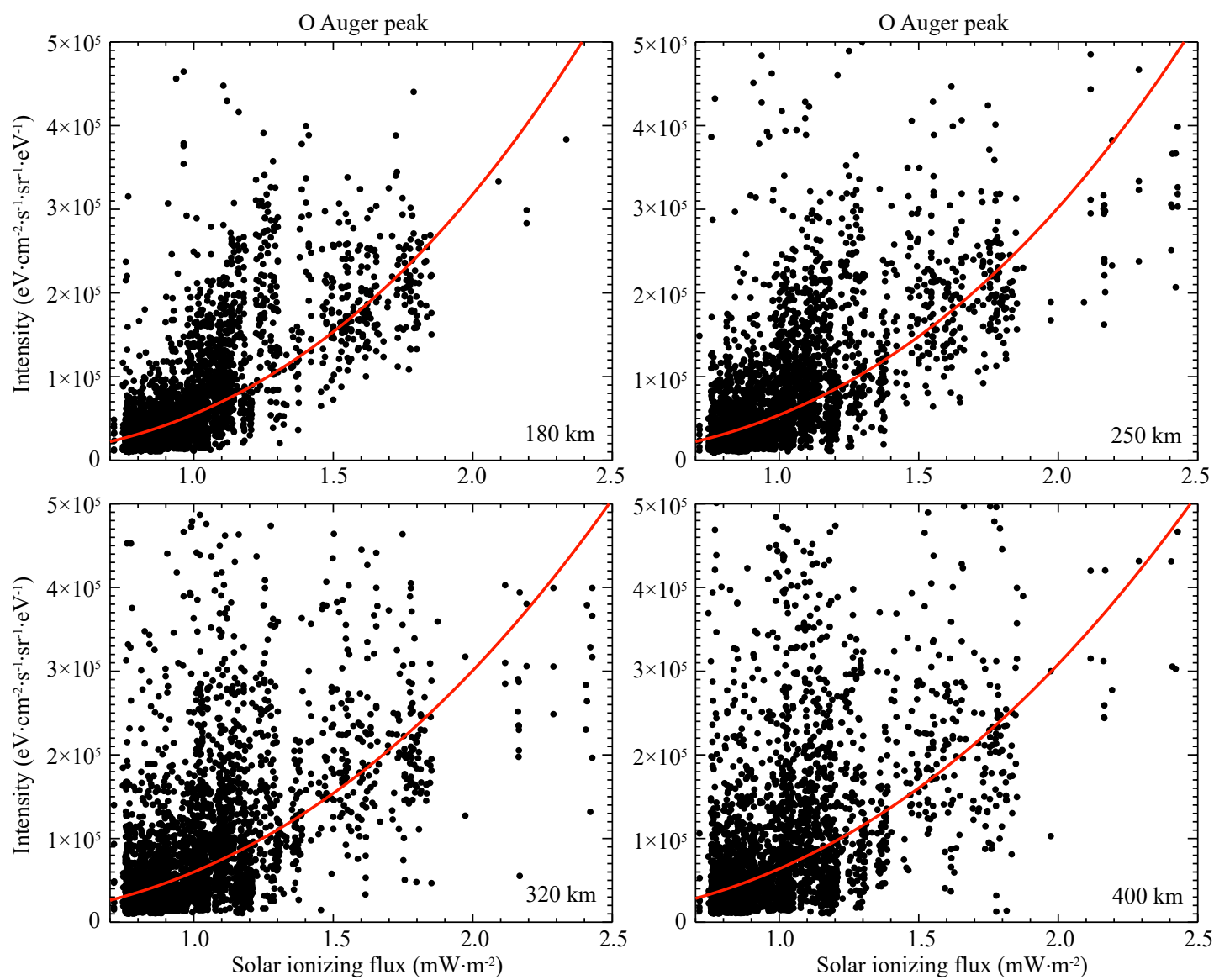

Figure 3. Similar to Figure 2 but for solar cycle variation of photoelectron intensity at the center of the O Auger peak.

Table 1. Best-fit power indexes for solar cycle variations of photoelectron intensity at the centers of the HeII and O Auger peaks (see text for details).

\begin{tabular}{ccc}
\hline \multirow{2}{*}{ Altitude $(\mathrm{km})$} & \multicolumn{2}{c}{ Best-fit power index, $a$} \\
\cline { 2 - 3 } & HeII peak & O Auger peak \\
\hline 180 & 1.21 & 2.62 \\
250 & 1.30 & 2.66 \\
320 & 1.27 & 2.53 \\
400 & 1.32 & 2.45 \\
Average & 1.28 & 2.57 \\
\hline
\end{tabular}

parameterized solar cycle variations presented here are well suited for future comparisons with kinetic model results obtained under different solar EUV/X-ray inputs (e.g. Liemohn et al., 2003, 2006; Xu SS et al., 2015; Xu SS and Liemohn, 2015; Sakai et al., 2015; Peterson et al., 2016).

\section{Acknowledgments}

This work is supported by the B-type Strategic Priority Program No. XDB41000000 funded by the Chinese Academy of Sciences and the pre-research project on Civil Aerospace Technologies No. D020105 funded by China's National Space Administration (CNSA). The authors also acknowledge support from the National Natural Science Foundation of China (NSFC) through grants
41904154, 41525015, and 41774186. The data used in this work are publicly available at the MAVEN Science Data Center (http://lasp. colorado.edu/maven/sdc/public/).

\section{References}

Adams, D., Xu, S., Mitchell, D. L., Lillis, R. J., Fillingim, M., Andersson, L., Fowler, C., Connerney, J. E. P., Espley, J., and Mazelle, C. (2018). Using magnetic topology to probe the sources of Mars' nightside ionosphere. Geophys. Res. Lett., 45(22), 12190-12197. https://doi.org/10.1029/2018GL080629

Cao, Y. T., Wellbrock, A., Coates, A. J., Caro-Carretero, R., Jones, G. H., Cui, J., Galand, M., and Dougherty, M. K. (2020a). Field-aligned photoelectron energy peaks at high altitude and on the nightside of Titan. J. Geophys. Res. Planets, 125(1), e06252. https://doi.org/10.1029/2019JE006252

Cao, Y. T., Cui, J., Wu, X. S., and Zhong, J. H. (2020b). Photoelectron pitch angle distribution near Mars and its implications on cross terminator magnetic field connectivity. Earth Planet. Phys., 4(1), 17-22. https://doi.org/10.26464/epp2020008

Coates, A. J., Tsang, S. M. E., Wellbrock, A., Frahm, R. A., Winningham, J. D., Barabash, S., Lundin, R., Young, D. T., and Crary, F. J. (2011). lonospheric photoelectrons: Comparing Venus, Earth, Mars and Titan. Planet. Space Sci, 59(10), 1019-1027. https://doi.org/10.1016/j.pss.2010.07.016

Coates, A. J., Wellbrock, A., Frahm, R. A., Winningham, J. D., Fedorov, A., Barabash, S., and Lundin, R. (2015). Distant ionospheric photoelectron energy peak observations at Venus. Planet. Space Sci., 113-114, 378-384. https://doi.org/10.1016/j.pss.2015.02.003

Cui, J., Wu, X. S., Xu, S. S., Wang, X. D., Wellbrock, A., Nordheim, T. A., Cao, Y. T., Wang, W. R., Sun, W. Q., ... Wu, S. Q. (2018). Ionization efficiency in the dayside Martian upper atmosphere. Astrophys. J. Lett., 857(2), L18. https://doi.org/10.3847/2041-8213/aabcc6 
Eparvier, F. G., Chamberlin, P. C., Woods, T. N., and Thiemann, E. M. B. (2015). The solar extreme ultraviolet monitor for MAVEN. Space Sci. Rev., 195(1-4), 293-301. https://doi.org/10.1007/s11214-015-0195-2

Frahm, R. A., Winningham, J. D., Sharber, J. R., Scherrer, J. R., Jeffers, S. J., Coates, A. J., Linder, D. R., Kataria, D. O., Lundin, R., ... Dierker, C. (2006a). Carbon dioxide photoelectron energy peaks at Mars. Icarus, 182(2), 371-382. https://doi.org/10.1016/j.icarus.2006.01.014

Frahm, R. A., Sharber, J. R., Winningham, J. D., Wurz, P., Liemohn, M. W., Kallio, E., Yamauchi, M., Lundin, R., Barabash, S., ... McKenna-Lawler, S. (2006b). Locations of atmospheric photoelectron energy peaks within the Mars environment. Space Sci. Rev., 126(1-4), 389-402. https://doi.org/10.1007/s11214-006-9119-5

Garnier, P., Steckiewicz, M., Mazelle, C., Xu, S., Mitchell, D., Holmberg, M. K. G., Halekas, J. S., Andersson, L., Brain, D. A., ... Jakosky, B. M. (2017). The Martian photoelectron boundary as seen by MAVEN. J. Geophys. Res. Space Phys., 122(10), 10472-10485. https://doi.org/10.1002/2017JA024497

Haider, S. A., and Mahajan, K. K. (2014). Lower and upper ionosphere of Mars. Space Sci. Rev., 182(1-4), 19-84. https://doi.org/10.1007/s11214-014-0058-2

Han, Q. Q., Fan, K., Cui, J., Wei, Y., Fraenz, M., Dubinin, E., Chai, L. H., Rong, Z. J., Wan, W. X., ... Connerney, J. E. P. (2019). The relationship between photoelectron boundary and steep electron density gradient on Mars: MAVEN observations. J. Geophys. Res. Space Phys., 124(10), 8015-8022. https://doi.org/10.1029/2019JA026739

Hantsch, M. H., and Bauer, S. J. (1990). Solar control of the Mars ionosphere. Planet. Space Sci., 38(4), 539-542. https://doi.org/10.1016/00320633(90)90146-H

Jakosky, B. M., Grebowsky, J. M., Luhmann, J. G., and Brain, D. A. (2015). Initial results from the MAVEN mission to Mars. Geophys. Res. Lett., 42(21), 8791-8802. https://doi.org/10.1002/2015GL065271

Liemohn, M. W., Mitchell, D. L., Nagy, A. F., Fox, J. L., Reimer, T. W., and Ma, Y. J. (2003). Comparisons of electron fluxes measured in the crustal fields at Mars by the MGS magnetometer/electron reflectometer instrument with a $B$ field-dependent transport code. J. Geophys. Res. Planets, 108(E12), 5134. https://doi.org/10.1029/2003JE002158

Liemohn, M. W., Frahm, R. A., Winningham, J. D., Ma, Y., Barabash, S., Lundin, R., Kozyra, J. U., Nagy, A. F., Bougher, S. M., ... Dierker, C. (2006). Numerical interpretation of high-altitude photoelectron observations. Icarus, 182(2), 383-395. https://doi.org/10.1016/j.icarus.2005.10.036

Mantas, G. P., and Hanson, W. B. (1979). Photoelectron fluxes in the Martian ionosphere. J. Geophys. Res. Space Phys., 84(A2), 369-385. https://doi.org/10.1029/JA084iA02p00369

Matta, M., Galand, M., Moore, L., Mendillo, M., and Withers, P. (2014). Numerical simulations of ion and electron temperatures in the ionosphere of Mars: Multiple ions and diurnal variations. Icarus, 227, 78-88. https://doi.org/10.1016/j.icarus.2013.09.006

McFadden, J. P., Kortmann, O., Curtis, D., Dalton, G., Johnson, G., Abiad, R., Sterling, R., Hatch, K., Berg, P., ... Jakosky, B. (2015). MAVEN SupraThermal and thermal ion compostion (STATIC) instrument. Space Sci. Rev., 195(1-4), 199-256. https://doi.org/10.1007/s11214-015-0175-6

Mitchell, D. L., Lin, R. P., Mazelle, C., Rème, H., Cloutier, P. A., Connerney, J. E. P., Acuña, M. H., and Ness, N. F. (2001). Probing Mars' crustal magnetic field and ionosphere with the MGS Electron Reflectometer. J. Geophys. Res. Planets, 106(10), 23419-23427. https://doi.org/10.1029/2000JE001435

Mitchell, D. L., Mazelle, C., Sauvaud, J. A., Thocaven, J. J., Rouzaud, J., Fedorov, A., Rouger, P., Toublanc, D., Taylor, E., ... Jakosky, B. M. (2016). The MAVEN solar wind electron analyzer. Space Sci. Rev., 200(1-4), 495-528. https://doi.org/10.1007/s11214-015-0232-1

Morgan, D. D., Gurnett, D. A., Kirchner, D. L., Fox, J. L., Nielsen, E., and Plaut, J. J. (2008). Variation of the Martian ionospheric electron density from Mars Express radar soundings. J. Geophys. Res. Space Phys., 113(A9), A09303. https://doi.org/10.1029/2008JA013313

Peterson, W. K., Thiemann, E. M. B., Eparvier, F. G., Andersson, L., Fowler, C. M., Larson, D., Mitchell, D., Mazelle, C., Fontenla, J., ... Jakosky, B. (2016).
Photoelectrons and solar ionizing radiation at Mars: Predictions versus MAVEN observations. J. Geophys. Res. Space Phys., 121(9), 8859-8870. https://doi.org/10.1002/2016JA022677

Sakai, S., Rahmati, A., Mitchell, D. L., Cravens, T. E., Bougher, S. W., Mazelle, C., Peterson, W. K., Eparvier, F. G., Fontenla, J. M., and Jakosky, B. M. (2015). Model insights into energetic photoelectrons measured at Mars by MAVEN. Geophys. Res. Lett., 42(21), 8894-8900. https://doi.org/10.1002/2015GL065169

Sakai, S., Andersson, L., Cravens, T. E., Mitchell, D. L., Mazelle, C., Rahmati, A., Fowler, C. M., Bougher, S. W., Thiemann, E. M. B., ... Jakosky, B. M. (2016). Electron energetics in the Martian dayside ionosphere: Model comparisons with MAVEN data. J. Geophys. Res. Space Phys., 121(7), 7049-7066. https://doi.org/10.1002/2016JA022782

Shutte, N. M., Király, P., Cravens, T. E., Dyachkov, A. V., Gombos, T. I., Gringuaz, K. I., Nagy, A. F., Sharp, W. E., Sheronova, S. M., ... Verigin, M. (1989). Observation of electron and ion fluxes in the vicinity of Mars with the HARP spectrometer. Nature, 341(6243), 614-616. https://doi.org/10.1038/341614a0

Thiemann, E. M. B., Chamberlin, P. C., Eparvier, F. G., Templeman, B., Woods, T. N., Bougher, S. W., and Jakosky, B. M. (2017). The MAVEN EUVM model of solar spectral irradiance variability at Mars: Algorithms and results. J. Geophys. Res. Space Phys., 122(3), 2748-2767. https://doi.org/10.1002/2016JA023512

Trantham, M., Liemohn, M., Mitchell, D., and Frank, J. (2011). Photoelectrons on closed crustal field lines at Mars. J. Geophys. Res. Space Phys., 116(A7), A07311. https://doi.org/10.1029/2010JA016231

Vogt, M. F., Withers, P., Mahaffy, P. R., Benna, M., Elrod, M. K., Halekas, J. S., Connerney, J. E. P., Espley, J. R., Mitchell, D. L., ... Jakosky, B. M. (2015). lonopause-like density gradients in the Martian ionosphere: A first look with MAVEN. Geophys. Res. Lett., 42(21), 8885-8893. https://doi.org/10.1002/2015GL065269

Withers, P. (2009). A review of observed variability in the dayside ionosphere of Mars. Adv. Space Res., 44(3), 277-307. https://doi.org/10.1016/j.asr.2009.04.027

Wu, X. S., Cui, J., Cao, Y. T., Liu, L. J., Zhou, Z. J., Huang, Y. Y., He, F., and Wei, Y. (2019a). On the hardness of the photoelectron energy spectrum near Mars. J. Geophys. Res. Planets, 124(11), 2745-2753. https://doi.org/10.1029/2019JE006093

Wu, X. S., Cui, J., Yu, J., Liu, L. J., and Zhou, Z. J. (2019b). Photoelectron balance in the dayside Martian upper atmosphere. Earth Planet. Phys., 3(5), 373-379. https://doi.org/10.26464/epp2019038

Xu, S. S., Liemohn, M. W., Peterson, W. K., Fontenla, J., and Chamberlin, P. (2015). Comparison of different solar irradiance models for the superthermal electron transport model for Mars. Planet. Space Sci., 119, 62-68. https://doi.org/10.1016/j.pss.2015.09.008

$\mathrm{Xu}$, S. S., and Liemohn, M. W. (2015). Superthermal electron transport model for Mars. Earth Space Sci., 2(3), 47-74. https://doi.org/10.1002/2014EA000043

Xu, S. S., Mitchell, D., Liemohn, M., Dong, C. F., Bougher, S., Fillingim, F., Lillis, R., McFadden, J., Mazelle, C., ... Jakosky, B. (2016a). Deep nightside photoelectron observations by MAVEN SWEA: Implications for Martian northern hemispheric magnetic topology and nightside ionosphere source. Geophys. Res. Lett., 43(17), 8876-8884. https://doi.org/10.1002/2016GL070527

Xu, S. S., Liemohn, M., Bougher, S., and Mitchell, D. (2016b). Martian highaltitude photoelectrons independent of solar zenith angle. J. Geophys. Res. Space Phys., 121(4), 3767-3780. https://doi.org/10.1002/2015JA022149

Xu, S. S., Mitchell, D., Liemohn, M., Fang, X. H., Ma, Y. J., Luhmann, J., Brain, D., Steckiewicz, M., Mazelle, C., ... Jakosky, B. (2017). Martian low-altitude magnetic topology deduced from MAVEN/SWEA observations. J. Geophys. Res. Space Phys., 122(2), 1831-1852. https://doi.org/10.1002/2016JA023467

Yao, M. J., Cui, J., Wu, X. S., Huang, Y. Y., and Wang, W. R. (2019). Variability of the Martian ionosphere from the MAVEN radio occultation science experiment. Earth Planet. Phys., 3(4), 283-289. https://doi.org/10.26464/epp2019029 